\title{
BDTice
}

PERTIGKS OF THIS REFORT ARE H.LECEBLE.

It has been reproduced trom the best available copy to germit the broatest possible availability.

MN ONHE

\section{FORIG: A Modification of the ORIGEN2 Isotope-Gerneration and Depletion Code for Fusion Problems}

\author{
James A. Blink
}

Manusrript date: March 3, 1982

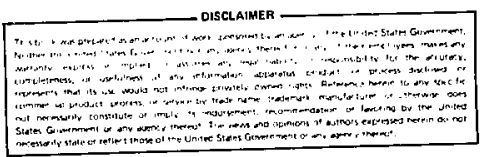

LAWRENCE LÏVERMORE LABORATORY University of California • Livermore, California 994550 


\section{Contents}

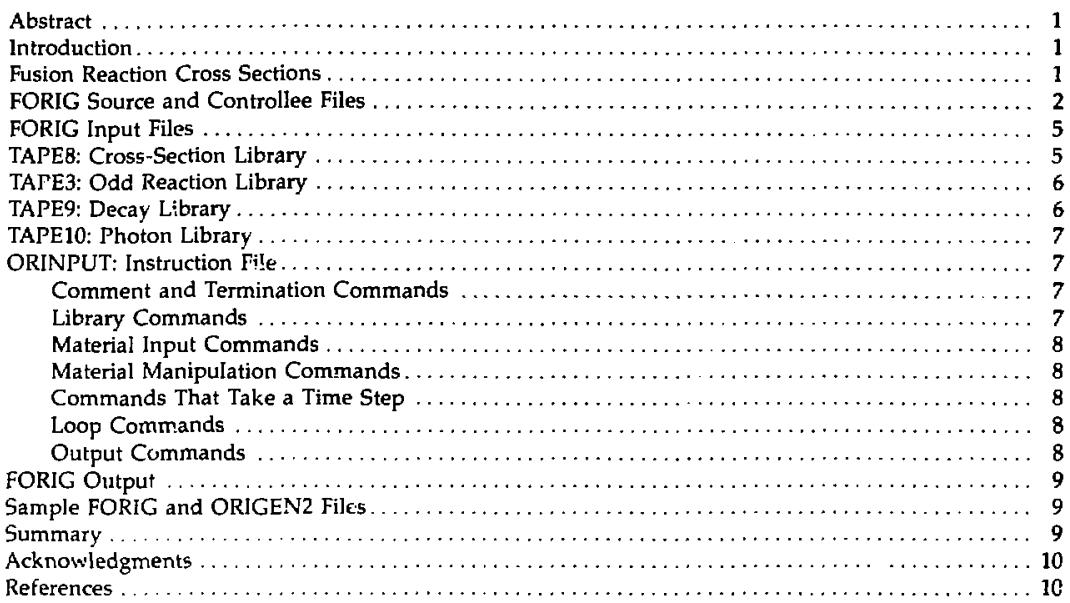




\title{
FORIG: A Modification of the ORIGEN2 Isotope-Generation and Depletion Code for Fusion Problems
}

\begin{abstract}
This report describes how to use the FORIG computer code to solve isotope-generation and depletion problems in fusion and fission reactors. FORIG is an adaptation of ORIGEN2 to run on a Cray-1 computer, and to accept more extensive activation cross sections.
\end{abstract}

\section{Introduction}

ORIGEN2 is a widely used computer corle for calculating the buildup, decay, and processing of radioactive materials in fission reactors. This :eport documents changes made in the ORIGEN2 code to permit use of an LLNL Cray-1 computer. With only slight changes in the input file, any of the standard fission problems can run at LLNL as they do at Oak Ridge and elsewhere. In addition, the corle has been modified to read activationcross-section libraries appropriate for iusion reactor design problems. If the flux-weighted cross sections are constant in timi, a single FORIG run can calculate activation and decay in a fusion reactor component. To address fission-fusion hybrid reactor designs, FORIG will require further modification to account for actiride burnup and fission product buildup. This may be done in a manner similar to the fission reactor treatment in ORIGEN2, or it may be accomplished by alternately using a neutron flux code, a cross-section collapsing code, and FORIG.

\section{Fusion Reaction Cross Sections}

The ORIGEN2 code cross-section library includes six standard reactions for non-fissionable nuclides:

$(n, 2 n)(n, 2 n) \operatorname{exc}(n, p)(n, \alpha)(n, \gamma)(n, \gamma)$ exc,

where "exc" indicates the daughter is in an excited state. Additional reactions may be added in a separate file called "odd reactions" in the ORIGEN2 manual. For fission reactors, there are few additionai reactions. However, fusion reactor problems require an odd reaction file many times larger than the standard file because of the higher-energy neutrons. Further, ORIGEN2 ignores helium ar.d hydrogen isotope production from odd reactions.

The neutron-energy spectrum varies between regions in a single fusion reactor design, and between designs. ORIGEN2 requires flux-averaged (i.e., energy-weighted) cross sections. Hence, multiple cross-section libraries are required for fusion reactors and even for different regions of any specific design. The ORI IB code ${ }^{2}$ has been written at LLNL to utilize the ACTL cross section library ${ }^{3}$ and the TART neutronics code ${ }^{4}$ to produce a one-energy-group cross section library for any region of a fusion reactor. The ORLIB output includes a cross section file with 11 standard reactions:

$$
\begin{aligned}
& (n, 2 n)(n, 2 n) \operatorname{exc}(n, 3 n)(n, n p)(n, n \alpha)(n, p) \\
& (n, d)(n, t)(n, \alpha)(n, \gamma)(n, \gamma) \text { exc. }
\end{aligned}
$$

ORLIB also produces an odd reaction file. Table 1 lists the 26 reaction types processed by the code. FORIG will accept these files if the library number is set $\geq 700$. If the library number is less than 700 , 
Table 1. Reaction types available from the ORLIB code.

\begin{tabular}{|c|c|c|c|}
\hline Type No. & Reaction & Type No. & Reaction \\
\hline 12 & $(n, 2 n)$ & 29 & $(n, 2 n p) a$ \\
\hline 13 & $(n, 3 n)$ & 30 & $(\mathrm{n}, \gamma \mathrm{n} \boldsymbol{\alpha})$ \\
\hline 14 & $(n, 4 n)$ & 31 & $(n, 2 n p \alpha) \alpha$ \\
\hline 15 & ( $n, f$ iss) & 37 & $(1,2 \alpha) \alpha$ \\
\hline 20 & $(\mathbf{n}, \mathbf{n p})$ & 38 & $\left(n,{ }^{3} \mathrm{He} \alpha\right) \alpha$ \\
\hline 21 & $(n, p n)$ & 39 & $(n, t p) \alpha$ \\
\hline 22 & (n,nd) & 40 & $(n, p)$ \\
\hline 23 & $(n, n d \alpha) \alpha$ & 41 & $(n, d)$ \\
\hline 24 & $(n, n t)$ & 42 & $(n, t)$ \\
\hline 25 & $\left(n, n^{3} \mathrm{He}\right)$ & 43 & $(n, t \alpha) \alpha$ \\
\hline 26 & $(\mathbf{n}, \mathbf{n} \alpha)$ & 44 & (n, $\left.{ }^{3} \mathrm{He}\right)$ \\
\hline 27 & $(n, n 2 \alpha) \alpha$ & 45 & $(n, a)$ \\
\hline 28 & $(n, n t \alpha) \alpha$ & 46 & $(n, \gamma)$ \\
\hline
\end{tabular}

the standard ORIGEN2 cross-section format is ac-. cepted. FORIG also calculates helium and hydrogen isotope production from both standard and odd reactions.

FORIG, like ORIGEN2, divides problems into three parts: light nuclides (also cailed activation products), actinides, and fission products. In some problems, a nuclide may appear in two categories, and the code will treat the two separately. In pure fusion problems, there are no actinides or fission products, and no libraries are input for these categories. In this case, zeros are entered in the apprupriate blocks in the LIB and PHO commands of the input file. (All the FORIG commands are discussed later in this report.)

\section{FORIG Source and Controllee Files}

The amount of memory required to solve a FORIG or ORIGEiv2 problem is deternined by the number of nuclides, cross sections, etc. To prevent excessive use of memory, the source file is divided into a small main program (FORIGM) and a large file containing all the subroutines required by the main program. The subroutines do not change from problem to problem, but the dimensions set up in FORIGM must be predetermined by the user. A listing of the main program is provided in Fig. 1. The figure also provides information required to set the dimensions of the arrays. Once this is done, the program can be compiled.

At LLNL, the appropriate files are available in J. Blink's take directory:

\section{XPORT / $t \mathrm{y}$}

.RD .084975:ORIGEN2 [FORIG FORIGM FCRIGMG FORIGS BFORIGS]

(XPORT is a file transport routine at LLNL).

FORIG is the controllee set up for a pure fusion problem with less than 800 nuclides in the decay library. If the problem of interest does not require actinides or fission products, and if the ex- isting decay library is adequate, then only FORIG need be read, and no compiling process is needed.

FORIGS is the file containing all the FORIG subroutines. This file is only required if the subroutines must be changed. Once the changes have been made, FORIGS is compiled into a library:

\section{RCFT I=FORIGS / $t v$}

The compiler, RCFT, produces the library file BFORIGS. If no changes are to be made in the subroutines, BFORIGS may be read directly from the take directory.

FORIGMG is the main program with generalized dimensions. After it is read and its dimensions adjusted, it may be renamed FORIGM and compiled:

RCFT I $=$ FORIGM, $\boldsymbol{X}=$ FORIG, LIB $=$ (BFORIGS,FORTLIB) $/ \mathrm{t}$ v.

The FORIGM file in the take directory is set up for a pure fusion problem with less than 800 nuclides. The controllee is named FORIG. 


\begin{tabular}{|c|c|c|}
\hline AAAA & (13) & No. ouput vectors \\
\hline BBBB & $(800)$ & Maximum No. nuclides \\
\hline $\operatorname{cccc}$ & (15) & $\begin{array}{l}\text { Maximum No. nonzero cross-section } \\
\text { and decay reactions per nuclide } \\
\text { (cannot exceed } 15 \text { without } \\
\text { redimensioning the subroutines) }\end{array}$ \\
\hline DDDD & $(2000)$ & Total No. nonzero matrix elements \\
\hline EEEE & (4) & No. nonzero fission-product yields \\
\hline FFFF & (4) & Maximum No. fission produets \\
\hline 8 & & Maximum No. actinides +1 \\
\hline
\end{tabular}

HII

JjJ]

KKKK

LLLL MMAM NNNN

PPPP
(2000) Maximum No, nonzero matrix elements for long-lived nuclides

(10) No. storage vectors

(300) No. nonzero natural abundsnces

(3300) No. nonzero photon yizlds

[800] Maximum No. light nuclides

(4) Maximum fio. variable multipliers (i., RMULV)

(3) No. actinides with both direct fission-product yields and a variable fission cross section (usually 3; can be 4 for plutonium-enriched thorium fuels)

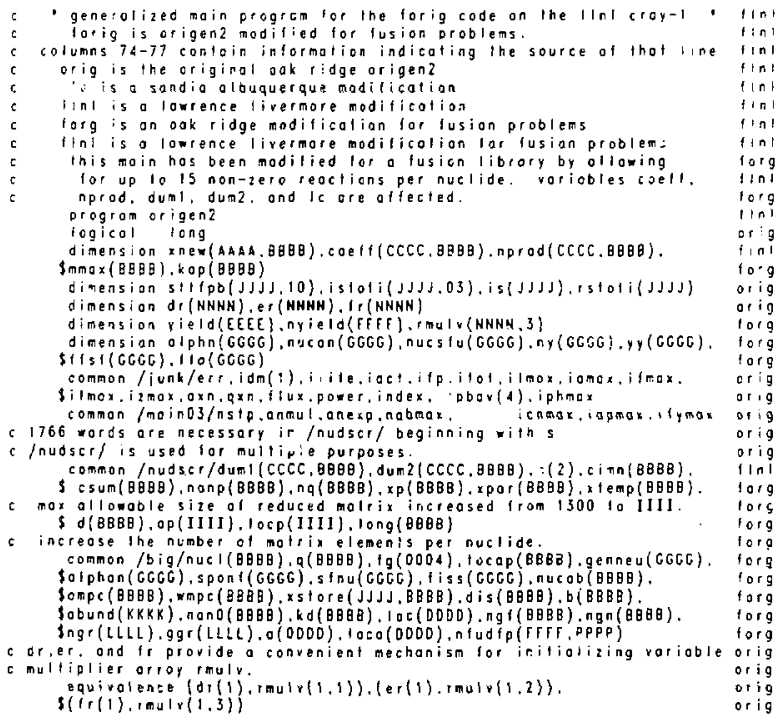

Figure 1. FORIG main source. The dimensions are set using the comments above the listing. Dimengions chosen for a pure fusion problem with less than $\mathbf{8 0 0}$ nuclides are listed parenthetically. 
[ these equiratences hove been realined. forg

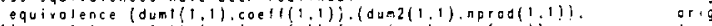

$\$(x \operatorname{lemp}(1), \operatorname{mox}(1)),(k \operatorname{kop}(1, d(1)) \cdot(\operatorname{xnew}(1,1), d u m 1(1,1))$ torg

equirolence (oo(i) clahn(1)). (otohn(GGCG). nucan(i)).(nucan(toto). forg

Investu(1)). (nuesiu(G:CG).ny(i)). (ny(GGGG).yy(1)).(yy(GGGG). forg

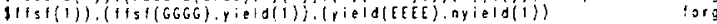
coll dropitie( itorig,

corinput is instructions. tapes crass sections. Iapeg decar librarr.

tapelo photon librory. and lapes add reaclians.

call open(5, orinost , 2, netr)

iflner.it. o) coll eril(i)

cali open(8., tape8. 2 . netr)

iftner. It. 0) coli exilil

cali apen(9. Topeg. 2.neri)

if(nere.11. o) eoli exitili)

coll open\{10."1opeto', 2, nerr

ilner il. ol call exil(i)

coll open (3. tope3. 2, nerr)

il(nerr, It, O) callexit(i)

c ocout is main autput. tape? is exil malerial campasitions.

c topeti is alternatamain oulpul. torst2 s taper contenls.

tapeis is tapel1 cantents lapels is debug and error inlo.

topelo is roriaole octinide cross section nlo. and lapeso

is a marking file.

call createlo, orout , 3, 200000)

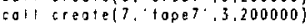

call create(11, 10pel1, 3,200000)

call $\operatorname{crerte}\left(122^{\circ}\right.$ tapel $\left.2^{\circ} .3,200000\right)$

$=211$ coole $(13, \cdot 10 p e 13 \cdot .3,200000)$

call creale(15. lopels.3.200000)

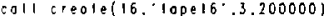

call creole $(50$. 'lope50.2.200000)

: inilialite poge counlet

nooge $=$ i Doge 0$\}$

roli nuodoc 6

$11=J j J J$

$m s=$ hAAA

I $c=C C C C$

i nox $=$ 4uHM

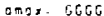

$1 \operatorname{lng} x=1615$

$1 \max =\mathrm{ag} \theta$

$7 \operatorname{mox}=0000$

ahm $0 x=L L L L$

opmax =III

if $f \operatorname{mox}=E E E E$

ก OO m S $x=K K X X$

icnmax =PPPP

$i d=F F F$

$10 \mathrm{~m}=\mathrm{NNHN}$

in

fin

$f|n|$

$f$ I $I$

I In I

I InI

||$n \mid$

I I $n$ t

$\ln \mid$

$|\ln |$

$|\ln |$

$|\ln |$

| In $\mid$

$f \ln 1$

$\ln \mid$

$\ln \mid$

$\ln 1$

$|\ln |$

$f|n|$

In

||$n \mid$

$|\ln |$

$\ln \mid$

I In I

I $|n|$

| In $\mid$

orig

orig

orig

or ig

orio

$|\mathrm{n}|$

forg

forg

lorg

lorg

lorg

lorg

forg

forg

forg

fla

forg

arig

c neutionsineution-: aduced lissior $0=$ thermol spectrum; $1=f a s t$ spectrum ortg $\eta \div 11=1$

nY $11=0$

$0, i g$

orig

c coll subroutine to reod card inpul from unit 5, print th on unil 6 , $b$ orig

c wile it on unit 50 . unit 50 is then tewaund and orient reads lhe orig

c doto trom unit 50 coll listit $(5,6,50)$

rewind 50

c mainl handtes the miscellaneous initialitation data

1 coti moinl(nytf, sfnu, olphn, nucon. nucsfu, ny. ry. onmul onexp)

c moin2 leads the origen2 commonds

2 call main2(nsip)

c moin 3 execules the origeriz commonos

3 coll moin3!

I Iong.sIIIpb,isloli, is, rgtoti, Ix, $m x$, lc, ifd,

inucab. nono,kd, Ioe, ngl, ngn, ngr, ny ie id, nonp, nq, locp, mnax, kop,

laco.nIudip. cimn.csun. $s$,

\$nuc1.9.19.10cop.genneu.olphon.spont.stau. fiss.ompc rmpe, kstore.

idis.b.ggr, yield.o xp, xpar, xtemp,d, ap, coeff, nprod, xnew,

bolphn, nuean, nuestu, ny, yy. fls $f, f$ to, obund, rmulp. lom

c this "go 10 " provides the mechonism for execuling muliple problems

c ithino single iob.

go $10(1,2,3,4)$, nstp

4 cantinue

stop 100

end

orig

orig

orig

orig

orig

orig

or ig

orig

arig

orig

orig

orig

orig

orig

orig

orig

orig

orig

or ig

orig

orig

Figure 1. (Continued) 


\section{FORIG Input Files}

FORIG requires the following input files:

TAPE8 - cross-section library

TAPE3 - odd reaction libra:y

TAPE9 - decay library

TAPE 10 - photon library

ORINPUT - instruction list.

These files are very similar to those used with ORIGEN2. The changes are as follows:

(1) In ORIGEN2, TAPE9 includes both the decay and cross-section libraries. For fission reactor problems, Oak Ridge provides them separately, and the user must merge them (or concatenate them. according to the ORIGEN2 manual). In FORIG, the two files are kept separate.

(2) When the library number $\geq 700$, FORIG expects TAPE 3 and TAPE8 to be in the ORLIB format.
(3) The first line of the instruction file is a new lire (not used in ORIGEN2). If the line contains a negative integer, FORIG will not print neutron sources from spontaneous fission, neutron-induced fission, or $(\alpha, n)$ reactions. These tabies are not applicable to pure fusion problems.

(4) The LIB command has been changed to accommodate the separate cross-section and decay library files.

(5) The ORIGEN2 code has a maximum of seven loss paths (by activation or decay) for each nuclide. Additional loss paths are omitted and the program continues after printing a warning. FORIG uses up to 15 loss paths and error-exits if this maximum is exceeded. In this case, either the cross-section files must be altered to reduce the paths, or both the sources (FORIGM and FORIGS) must be redimensioned and recompiled.

(6) ORIGEN2 reads 80 -character lines, FOR'S reads 120 -character lines.

\section{TAPE8: Cross-Section Library}

If the library number is $<700$, the standard ORIGEN2 library is used:

NLB TITLE (For the light nuclide segment) NLB ZZAAAI Sn $\gamma$ Sn2n Sn $\alpha$ Snp Sn $\gamma x$ $\operatorname{Sn2nx}-1.0$
-1

NLB TITLE (For the actinide segment)

NLB ZZAAAI $\operatorname{Sn} \gamma \operatorname{Sn} 2 n \operatorname{Sn} 3 n$ Snf Sn $\gamma x$ $\operatorname{Sn} 2 n x-1.0$

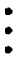

$-1$

NLB TITLE (For the fission product segment)

NLB ZZAAAI Sn $\gamma$ Sn2n Sn $\alpha$ Snp Sn $\gamma x$ $\operatorname{Sn} 2 \mathrm{n} \times 1.0$

$Y(1) Y(2) Y(3) Y(4) Y(5) Y(6) Y(7) Y(8)$

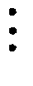

$-1$

where

NLB is the library number for each segment; ZZAAAI is the nuclide identification;

$\operatorname{Sn} \gamma$ is the cross section for the $(n, \gamma)$ reaction;
Sn2nx is the cross section for the $(n, 2 n)$ reaction leaving an excited daughter;

$\mathbf{Y}(1)$ through $\mathbf{Y}(8)$ are the fission product yield in percent from up to 8 actinides as specified in Table 5.2 of the ORIGEN2 manual. The $Y(1)$ through $Y(8)$ line is omitted if the previous line ends in a ncgative number.

If the light nuclide library number is $\geq 700$, the ORLIB format is used. For a pure fuision problem,

NLB TITLE

Comment line

ZZAAAI Sn2n Sn2nx Sn3n Snnp Snno Snp Snd Snt Sn $\alpha$ Sn $\gamma$ Sn $\gamma x$

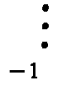

This example includes no cross sections for actinides or fission products. If the problem includes either, they are entered in the original ORIGEN2 format following the light nuclide segment.

In both formats, the code will error exit if cross sections are entered for nuclides not in the appropriate decay-library segment. 


\section{TAPE3: Odd Reaction Library}

If the light nuclide library number is $<700$, the odd reactions are added in the ORIGEN2 format

\section{ZAAAI ZZAAAI SIGMA

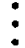

where the tirst and second entries on each line are the parent and daughter nuclide identifications. When this format is uscd, hydrogen and helium isotope production are not followed for the odd reactions.

If the light nuclide library number is $\geq 700$, the odd reactions are added in the ORLIB format:

NLIB TITLE

Comment Line
ZZAAAI ZZAAAI SIGMA TYPE Z?AAAI ZZAAAI SIGMA TYPE

-

where TYPE is the reaction type number (Table 1). With this format, hydrogen and helium isotope production is follo:ved for both the odd and the standard reactions.

In either format, each odd reaction is added to the first library segment (light nuclide, actinide, fission product) that includes the parent in the decay library. Also, if replacement decay or cross section lines are to be inserted in any of the six library segments, these lines precede the odd reaction formats shown above. (The LIB and LPU commands force the code to read each line in its proper format, as is discussed later.)

\section{TAPE9: Decay L.ibrary}

This library is input in the ORIGEN2 format.

NLB TITLE (For the light nuclide segment)

NLB ZZAAAI IU THALF F $\beta \times$ F $\beta+$ +EC $F \beta+E C \times F \alpha$ Fit

NLB Fsf Fn $\beta$ QREC ABUND ARCG WRCG

$-1$

NLIB TITLE (For the actinide segment)

$-1$

NLIB TITLE (For the fission product segment)$$
-1
$$

where

IU indicates the time units for the half life, THALF;

$\mathbf{F} \boldsymbol{\beta} \mathbf{x}$ is the fraction of all decays by negatron (beta) emission with an excited daughter;

$F \beta+E C$ is the fraction of all decays by positron emission or electron capture;
$\mathrm{F} \beta+\mathrm{EC} x$ is the fraction of all decays by positron emission or electron capture with an excited daughter;

$F \boldsymbol{\alpha}$ is the fraction of all decays by alpha emission;

Fit is the fraction of all decays by internal transition;

Fsf is the fraction of all decays by spontaneous fission;

$\mathrm{Fn} \beta$ is the fraction of all decays by (neutron plus beta) decay;

QREC is the total recoverable energy (not including neutrinos) released per decay, in $\mathrm{MeV}$;

ABUND is the natural isotopic abundance;

$A R C G$ is the radioactivity concentration guide (RCG) for continuous inhalation in unrestricted areas as given in Table II, Column I, 10CFR20 using the lower of the soluble and insoluble values, and

WRCG is the RCG for continuous ingestion from Column II of the same table in 10CFR 20 .

The fraction of negatron beta decays with a ground state daughter is calculated irom the above fractions. Since the decay library is used to produce the nuclide list for the calculation, both radioactive and stable nuclides of interest must be includr.a. 


\section{TAPE10: Photon Library}

This library is input in the ORIGEN2 format.

NLB TITLE (For the light nuclide segment) $\operatorname{RPH}(J)$

NLB ZZAAAI NGP(1) RPH(1) • • NGP(j)

NGP(J+1) RPH(J+1) . - NGP(18)

RPH(18) -1 where

NGP is the group number, a: d

RPH is the photon intensity per disintegration.

Only nonzero entries need be made. If actinides and fission products are used in the problem, their segments should be added to the example above.

\section{ORINPUT: Instruction File}

The input begins with four lines, each usually consisting of " -1 ." The negative entries suppress neutron source printing, decline to replace default individual element fractional recovery efficiencies, decline to replace default element group fractional recovery efficiencies, and decline to replace element group membership assignments.

The file continues with the individual commands, which are listed below by category. More detailed explanations are available in the ORIGEN2 manual.

\section{Comment and Termination}

\section{Commands}

RDA prints comments in the command list. (Comments may also be added at the end of most command lines.)

TIT specifies the title to be printed on the output table pages.

BAS prints a comment line on the output table pages. For example, the comment can indicate that the calculation is based on one metric tun of steel in the reactor.

HED allows a time column heading to be changed from the actual time to a 10 character alphanumeric heading.

STP allows a new case to begin with new commands, libraries, and/or initialization data.

END terminates execution.

\section{Library Commands}

LIB inperts the decay and cross section libraries. The format of this command has been one additional input. The FORIG format is

\section{LIB NLIB(1) * * NLIB(13)}

where

NLIB(1) is $>0$ if printing of the reaction rate matrix is desired (i.e., the production rate from each parent, the decay rate, and the removal rate by activation are printed for each nuclide);

NLIB(2) is the light nuclide decay library number;

NLIB(3-4) are the actinide and fission product decay library numbers ( 0 for pure fusion problems);

NLIB(5-7) are the three cross section libiery numbers; (Note: If a decay or cross-section library number is negative, replacement cards will also be read later for that library.)

NLIB(8) is the input unit number for the cross seciion library file (use 8 ):

NLIB(9) is the input unit number for the decay library file (use 9);

NLIB(10) is the input unit number for the replacement decay and cross-section lines, and for reading odd reactions (use 3);

NLIB(11) is the number of odd reactions to be read. If $N L I B(11)$ is negative, the read will be on unit NLIB(10). If NLIB(11) is positive, the read will be on unit $\mathrm{NLIB}(8)$. Ust negative NLIB(11).

NLIB(12) dstermines the set of actinides having direct fission product yields (use $\mathbf{0}$ for fusion problems); and

NLIB(13) determines the set of variable actinide cross sections to be used (use 0 for fusion problems). 
I.PU identifies nuclides requiring replacement lines in decay or cross section library segments. One LPU command must precede the LIB command for each negative library number in the L.IB command.

PHO inputs the photon libraries.

LIP determines whether the decay, crosssection, and photon libraries are printed. The LIP command must precede the LIB command.

\section{Material Input Commands}

INP forces the code to read the input material comporitions. The program is set up to read the compositions at the end of the input command file (ORINPUT). However, a previous FORIG run could have produced an output file in the froper format for input to this problem. If this file is to be used directly, it should be named "TAPE4," and "TAPE4" should be opened in the MAIN program. The INP command also allows input of continuous feed rates and continuous removal rates. The data for these rates follows the initial composition input at the end of ORINPUT.

WAC allows materiai to be continuously removed from the reactor with element dependent efficiency. This removed waste can then be decayed.

PRO allows a material, be processed at a given time into two segments with element dependent efficiency.

\section{Material Manipulation Commands}

FORIG maintains the material composition as a vector at each time step. Since only those vectors required for output or subsequent calculation need be kept, it is sometimes convenient to manipulate the vectors.

MOV allows a material composition to be moved from one vector to another. The command allows a constant nultiplier to be applied to the composition. The heading is also moved.
ADD allows addition of one material to a second material. The command also allows the material being added to be multiplied by a constant prior to the addition The heading for the first vector will be moved to the second vector with this command.

KEQ allows two materials to be blended until the resultant material has an infinite multiplication factor that matches either a third material or an input value.

FAC calculates the sum, product, or ratio of the total fission product plus actinide mass of iwo vectors or of a vector and an input constant. The resulting constant factor can then be used as a multiplier in subsequent ADD or MOV commands.

\section{Commands That Take \\ a Time Step}

DEC allows a composition vector to decay for a specified time.

IRF allows activation and decay of a composition vector in a constant neutron flux for a specified time.

IRP allows activation and decay of a composition vector at a constant power for a specific time (only applies to fission problems).

BUP calculates the average burnup, flux, and specific power for an irradiation period (only applies to íssion problems).

\section{Loop Commands}

EOL sets up a do loop through a set of instructions.

CON ends a do loop.

REC counts the number of times a do loop has been executed (for printing in the output tables).

\section{Output Commands}

OUT specifies composition vectors to be printed.

OPTL specifies output data to be printed for light nuclides for each vector in the OU' $\mathrm{I}$ command (options include composition, 
activity, thermal decay power, inhalation and ingestion biological hazard potential, chemical ingestion hazard, neutron absorption rate, neutron induced fission rate, $\alpha$ radioactivity, $\alpha$-induced neutron production, spontaneous fission neutron production, and photon emission rate). The OPTL command also specifies the units of the various tables and whether the output will be by nuclide, element, and/or in a condensed format. The OPTL command must precede the OUT command.
OPTA same as OPTL for actinides.

OPTF same as OPTL for fission products.

CUT defines cutoff limits for condensed output tables. If the output for a particular nuclide is less than the cutoff fraction times the total output at that time step, and if this is true for each time step being output, then the nuclide is omitted.

PCH creates an output file containing the material ccmpcsition in a format readable by future FOR!G calculations.

\section{FORIG Output}

The majority of the output is written in a file named OROUT (called TAPE6 in the ORIGEN2 manual). A table of contents for OROU' $\Gamma$ is written in file TAPE12. The OUT command can also specify sending output to a second file (TAPE11 with table of contents TAPE13); this is handy when an early, condensed haid-copy file is desired with a fuli microfiche output to foliow.
TAPE7 is an optional output composition file written by the PCH command. TAPE15 contains debug information and error messages. TAPE16 contains variab a actiride cross section information. Finally, TAPE50 is a working file set up and used by FORIG.

\section{Sample FORIG and ORIGEN2 Files}

At LLNI. I. Blink's take directory contains a number of FORIC, ORLIB, and ORIGEN2 files:

CONTENTS is a directory table of contents. ORLIB, ORLIBS, EORLIBIN, AC820312 and ADEX8203, are ORLIB files (see Ref. 2).

FORIGS, BFORIGS, FORIGMG, FORIGM, and FORIG are FORIG source and controllee files.

EORINPUT, ETAPE3, ETAPE8, ETAP'E9, and ETAPE 10 are example input and data files for a FORIG problem.

OR01C is the table of contents for the Oak Ridge supplied file list for ORIGEN2.

OR03C-OR18C are sample ORIGEN2 main pringrams for various fission reactors. OR03C has been modified to run on FORIG.

OP.IIC is the ORIGEN2 decay library.

OR22C-OR44C are the ORIGEN2 rross-section libraries for various fission reactors.

OR45C is the ORICEN2 photon library for $\mathrm{UO}_{2}$ Bremsstrahlung.

OR46C is the ORIGEN2 photon library for $\mathrm{H}_{2} \mathrm{O}$ Bremsstrahlung.

OR47C is the ORIGEN2 photon library for vacuum (no Bremsstrahlung).

OR48C-OR54C are sample ORIGEN2 output files for a single firsion reactor problem (TAPES 6 . $7,11,12,13,15$, and 16).

\section{Summary}

The ORIGEN 2 code has been modified to run at LLNL on a Cray-1 computer. The modified code, FORIG, will accept cross sections in both the ORIGEN2 format and the ORLIB format. The expanded cross section library available through
ORLIB permits use of FORIG to directly treat a wide variety of pure fusion reactor geometries. By iterating between a flux code, ORLIB, and FORIG, fusion-fission hybrid reactor geometries can also be handled. 


\section{Acknowledgments}

The author is grateful for helpful discussions with J. Hovingh, R. Howertun, G. Lasche, W. Meier, and M. Monsler of LLFL and D. Berwald of TRW. Considerable programming assistance from W. Warren, R. Dye, and the LLNL computer consulting office is appreciated. Finally, the author is indebted to A. Croff of ORNL for his patience in explaining many of the intricac: $s$ of ORIGEN?.

\section{References}

1. A. G. Croff, A User's Manual for the ORIGEN2 Computer Code, Oak Ridge National Laboratory, Gak Ridge, TN, ORNL/TM-7175 (1980). Also published as Radiation Shielding Information Center report, CCC-371.

2. J. A. Blink, R. E. Dye, and J. R. Kimlinger, ORLIB: A Computer Code that Produces One-Energy Group, Time- and Spatially-Avcraged Neutron Cross Seciions, Lawrence Livermore National Laboratory, Livermore, CA, UCRL-53262 (1981).

3. M. A. Gardner and R. I. Howerton, ACTL: Evaluated Neutron Activation Cross Section Library-Evaluation Techniques and Reaction Index, Lawrence Livermore National Laboratory, Livermore, CA, UCRL-50400, Vol. 18 (Oct. 17, 1978). In 1981, R. Howerton expanded the AC [L library to allow treatment of fusion reactor structural materials.

4. E. F. Plechaty and J. R. Kimlinger, TART_ 'P: A Coupled Neutron-Photor: Monte Carlo Transport Code, Lawrence Livermore National Laboratory, Livermoże, CA, UCRL-50400, Vol. 14 (July 4, 1976). 